Mastery of Surgery, $4^{\text {th }}$ Edition. Editors : RJ Baker, JE Fischer. Publishers : Lippincott Williams \& Wilkins, Philadelphia, 2001. Pages 2223. Price Rs 12,000/- ISBN 0-7817-2380-0.

T This popular book has been substantially revised and updated since the last edition seven years ago. Most of the chapters from previous edition have been extensively upgraded or rewritten, and several new chapters have been added. The handsome two-volume set consists of 206 chapters divided into nine sections starting from general topics like Preoperative Care and Basic Surgical Skills, and proceeding on to regional surgery. This multi-author book has contributions from 343 renowned surgeons, mainly from North America though eminent surgeons from 15 other countries have also made useful contributions.

The new edition incorporates the revolution of minimally invasive surgery very nicely. Common laparoscopic procedures like lap appendectomy, cholecystectomy and hemia repair have been described in detail; even relatively infrequent procedures like laparoscopic highly selective vagotomy. pelvic and retroperitoneal lymph node dissection have been included. A chapter on Minimally Invasive Vascular Surgery (endovascular surgery) describes angioplasty and stenting of peripheral arteries in some detail.

Most sections/chapters start with a description of relevant anatomy, so vital for performing good surgery. Details like preoperative preparation, positioning of the patient and assistants during surgery have been included in description of operations. Newer concepts and procedures like sentinel lymph node biopsy in breast cancer have also been included in the book. Some topics (eg. Echinococcal Cysts) have chapters by two different authors presenting a diametrically different approach to management of the disease. Infrequently performed procedures like splanchnic denervation of pancreas for intractable pain, and management of sequel of surgery like post-gastrectomy and post-vagotomy syndromes have also been described. The chapters are well supported by radiographs, excellent illustrations and diagrammatic concepts (eg. types of breast calcification, on page 577). Some practical problems like management of rectal foreign bodies have also been addressed.

One of the unique features, as also a major strength, is the editorial comment at the end of each chapter. Il places the topic in correct perspective. discusses the merits and indications of therapeutic options available (eg. trunsthoracic versus transabdominal fundoplication for reflux), cover important aspects not addressed by the author (eg. Visick grading after gastric surgery, classification of hepatorenal syndrome etc.). In a welcome move, these editorial comments are supported by recent journal references on the topic.

One of the shortcomings is that while regional trauma is adequately dealt with in the relevant chapters, there is no "general chapter' on trauma which could guide the surgeon on detailed patient assessment (including various trauma scores, which are mentioned only in passing), fixing priorities and sequence of investigations and management. Similarly, while trauma to various abdominal organs is discussed in considerable detail, there is no independent chapter on 'abdominal trauma' which could assist the surgeon on how to proceed in managing blunt (or for that matter penetrating) abdominal trauma, and the sequence of actions during exploratory laparotomy for this condition. The technique of tracheostomy is not described. I firmly believe that all surgeons should be familiar with this life-saving procedure; this could easily have been included in the chapter of basic surgical skills. Similarly stent-grafting of abdominal aortic aneurysms (AAA) finds only a brief mention at a time when over 40,000 such procedures have been performed world-wide, and the technique has revolutionised the way AAAs are now treated. From the Indian point of view a chapter on carcinoma of gall bladder would have been welcome. A couple of minor points. In the prophylaxis for deep vein thrombosis (page 38, and Table-9) : there is no mention of low molecular weight heparins, which are currently considered by the majority to be the standard treatment. Similarly, the choice of cefazolin sodium as the antimicrobial of choice (page 149. Table - 2) for all surgical prophylaxis appears odd, to say the least.

Notwithstanding the above minor deficiencies. the book is strongly recommended as essential reading for all surgical residents in training. It will also be a extremely useful companion for practising surgeons, irrespective of the fact whether he aspires to 'master' the art and science of surgery or not. It is also a mandatory requirement for library of a Medical College, as well as for the Zonal hospitals of Armed Forces.

\section{Coniributed by:-}

\section{Lt Col KM RAI SM}

Classified Specialist (Surgery \& Vascular Surgery), Army Hospital (Research \& Refertal), Delhi Cantt - 110010. 